\title{
Serum and Gene Expression Profile of Cytokines Following Combination of Yoga Training and Vitamin D Supplementation in Breast Cancer Survivors
}

\author{
Mehdi Naderi \\ Kharazmi University \\ Hajar Kordestani \\ Kharazmi University \\ Zahra Sahebi \\ Kharazmi University \\ Vahid Khedmati-zareh \\ Kharazmi University
}

Sadegh Amani-Shalamzari ( $\nabla$ amani_sadegh@khu.ac.ir)

Kharazmi University https://orcid.org/0000-0002-3021-8970

Mojtaba Kaviani

Acadia University

Joachim Wiskemann

Heidelberg University

Mahdieh Molanouri Shamsi

Tarbiat Modares University

Research article

Keywords: Hatha yoga, handgrip strength, quality of life, pro-inflammatory cytokines

Posted Date: June 16th, 2021

DOI: https://doi.org/10.21203/rs.3.rs-612814/v1

License: (c) (i) This work is licensed under a Creative Commons Attribution 4.0 International License. Read Full License 


\section{Abstract \\ Background}

This study aimed to examine the effect of a combination of yoga training with high vitamin $D$ dose supplementation on expression and systemic levels of inflammatory cytokines and psychophysical status of breast cancer survivors.

\section{Methods}

Thirty volunteered breast cancer survivors ( $48 \pm 8$ yrs.) were randomly allocated to a high dose (4000 IU) of vitamin $D$ supplementation (HD) group $(n=10)$, yoga with a high dose of vitamin $D$ (YHD) group $(n=$ $10)$, and yoga with a low dose (2000 IU) of vitamin D (YLD) group $(n=10)$. Participants performed the Hatha yoga style for 12 weeks, twice a week. Blood samples, quality of life (Qol) questionnaire, and physical performance tests were taken before and after the intervention

\section{Results}

Body fat percentage $(\eta p 2=0.36)$, handgrip strength $(\eta p 2=0.41)$, and Qol indicators include global health $(\eta p 2=0.54)$, functional scales $(\eta p 2=0.49)$, and symptoms scales $(\eta p 2=0.50)$ were significantly improved in the both YHD and YLD groups compared to the HD group $(p<0.05)$. Also, interleukin-10 (IL10) levels were markedly increased in the Y-HVD group compared to the Y-LVD and HVD groups. Moreover, there were significant decreases in tumor necrosis factor- $a$ (TNF- $a$ ) and interleukin- 6 levels in the Y-HVD group after the intervention. The anti-inflammatory index (IL-10/TNF-a) was significantly increased in both the yoga groups $(P<0.05)$.

\section{Conclusion}

Yoga promotes physical and psychological fitness and, in combination with a high dose of vitamin D, improves the cytokine profile, which can effectively manage the side effects associated with cancer.

\section{Introduction}

Breast cancer (BC) and treatments have several side effects on psychological and physical health, leading to a decline in the quality of life (Qol). The side effects could reduce survivors' muscle strength and aerobic capacity ${ }^{1}$ and expose patients to psychiatric disorders like depression and anxiety ${ }^{2}$. Fatigue is a common side effect of cancer treatment associated with decreased physical activity, which subsequently reduces performance abilities. Some documents link fatigue to elevated pro-inflammatory cytokines in cancer survivors ${ }^{3,4}$. In particular, serum levels of interleukin-6 (IL-6) ${ }^{5}$ and tumor necrosis 
factor- $a(T N F-a)^{6}$ may be increased as part of the host response to tissue damage or cancer treatments. Also, IL-10 is considered an anti-inflammatory cytokine; however, a dual role of IL-10 in breast cancer development was reported ${ }^{7}$. The IL-10/TNF-a ratio is widely used as an anti-inflammatory status ${ }^{8}$ and metabolic diseases ${ }^{9}$; thus, this ratio would be a better indicator of the treatment process. However, peripheral blood mononuclear cells (PBMCs) are considered the primary source of pre-and antiinflammatory cytokines; they are involved in changes in serum cytokine profiles ${ }^{10}$. It seems that investigating inflammatory markers-related gene expression in PBMCs may predict their changes in different cancer stages more accurately ${ }^{11}$. It is suggested that behavioral modifications such as exercise and a healthy diet are effective in regulating cytokine balance and managing adverse side effects.

Most BC patients and survivors, especially at menopause, have deficiencies in nutritional indicators, including vitamin $D(V D)$. Vitamin $D$ deficiency is associated with a decline in QoL ${ }^{12}$, and worsens cancer prognosis and increases the mortality rate in cancer patients ${ }^{13,14}$. However, more studies are needed on the VD doses that can be used to improve the QoL, especially in cancer survivors. Immune regulatory effects of VD supplementation particularly in high doses was observed in some studies ${ }^{15,16}$. Vitamin $D$ supplementation could lead to a shift from a Th1 to a Th2 phenotype ${ }^{17}$, and enhance IL-10 gene expression in T cells ${ }^{18}$, which in turn inhibits the production of pro-inflammatory cytokines. However, a systematic review proposed that possible effects of VD supplementation can induce auto-immunity effects with increasing regulatory $T$ activity and suppressing Th17 responses ${ }^{19}$. In contrast, a systematic review of randomized controlled trials appeared that a high dose of VD supplementation does not significantly change the serum levels of TNF- $a$ and IL- 6 in type 2 diabetes patients ${ }^{20}$. In addition, an animal study has reported high but not average-dose VD had caused mild hypercalcemia, which made T cells susceptible to pro-inflammatory activation ${ }^{21}$.

Exercise training is a behavioral modification to alleviate the side effects of cancer and its treatments. Yoga is a body-mind exercise that combines physical, mental, and spirit to improve psychological and physical health. Hatha yoga, the most common style executed in therapeutic settings, includes physical exercises (Asanas), breathing techniques (Pranayama), and meditation (Dyana) ${ }^{22,23}$. A systematic review reported that chronic stress via dysfunction in the classic neuroendocrine system and the sympathetic nervous system could induce tumorigenesis and promote cancer development ${ }^{24}$. Thus, stress management is crucial for cancer patients. Although the results are contradictory, it has been shown that yoga dampens inflammatory markers ${ }^{25}$, stress and anxiety ${ }^{26,27}$, and fatigue ${ }^{28}$ in cancer survivors; hence, yoga is an appropriate approach to improving the quality of life (QoL) of women with breast cancer. Also, some studies showed that yoga can have regulatory effect on nervous system ${ }^{23}$. Declined sympathetic nervous system tone ${ }^{23,29}$ and increased vagal activity ${ }^{29}$ are of mechanistic factors observed in yoga's benefits, both of which are involved in favorable endocrine and immune system changes that could lower inflammation markers. Therefore, in addition to improving physical and psychological fitness, yoga therapies could reduce inflammatory responses ${ }^{30}$. 
Recent studies have suggested the possible involvement of exercise training in managing high doses of selenium supplementation in breast cancer tumors. It is suggested that combination of exercise training and antioxidant supplementation in high doses can be effective on anti-tumor immunology and inflammatory cytokines ${ }^{31,32}$. However, considering the immune system's changes, the simultaneous effect of exercise training and VD intake has not been fully elucidated. Overall, given that a high dose of VD is safe for women at higher risk of $B C^{33}$ and recommended for $B C$ survivors to reduce inflammatory markers, yoga also plays an influential role in this process; we hypothesized that combining yoga exercise training and high VD dose can be more effective on expression and systemic levels of inflammatory cytokines. For approving our hypothesis, the effects of the combination of low VD dose and yoga exercise training on inflammatory cytokines were assessed in the current study. Moreover, inflammatory responses were associated with the psychological situation of breast cancer. Here, we assessed the possible relation between psychological indices and inflammatory expression in PBMCs and systemic levels of inflammatory cytokines in BC survivors.

\section{Methods}

\section{The experimental approach to the problem}

This study aimed to determine the effectiveness of 12 weeks of yoga training with the supplementation of VD on Qol and inflammatory markers in BC survivors. This study was a randomized, controlled trial with pre and post-tests. A few oncologists introduced eligible subjects to participate in the study. Initially, based on the initial level of VD, the subjects were divided into three groups randomly by a third person who was not in the research group. Pre- and post-intervention, Qol questioner, and handgrip strength tests were taken by the third assessor. In addition, blood sampling was taken, and circulatory levels of IL-6, IL10, and TNF- $a$ and their gene expressions in leukocytes were measured by a specific Elisa kit.

\section{Participants}

After the oncologists were informed of the research objectives, they introduced the subjects. Inclusion criteria were completed chemotherapy and radiotherapy, not have any acute medical disorders (cardiovascular diseases, diabetes), and not have any orthopedic conditions. The sample size was calculated by using G*Power Software version 3.1.9.6 (Düsseldorf, Germany). The estimated number of patients needed to assume a rejection criterion of 0.05 and 0.85 (1-beta) power, and a large effect $(f=0.65)$, was 10 persons per group, depending on the statistical test used. Thirty-three BC survivors who met inclusion criteria volunteered to participate in the study, but the data of 30 participants (age: $47.90 \pm$ 7.95 years; height: $160.93 \pm 6.12 \mathrm{~cm}$, body mass: $72.62 \pm 11.72 \mathrm{~kg}$ ) were obtained and analyzed finally. Exclusion criteria were getting worse a medical situation $(n=1)$, do not participate in more than four consecutive training sessions $(n=1)$, not interested in continue intervention, not complete the post-test $(n=1)$, and the physician would diagnose she must withdraw from the study. The third person randomly divided participants into a high dose (4000 IU) of VD supplementation (HVD) group ( $n=10)$, yoga with a high dose (4000 IU) of VD (Y-HVD) group ( $n=10)$, and yoga with a low dose (2000 IU) of VD (Y-LVD) group 
$(n=10)$. It seems that we needed a group that only practices yoga, but all cancer survivors consume different doses of VD, so due to ethical reasons, we could not put that group.

\section{Measurements}

\section{Physical Measurements}

All measurements were conducted by a third person who was not in our research team. By a height scale (Seca 206, Hamburg, Germany) and a digital body weight scale (Seca 803, Hamburg, Germany), height and body mass were measured, respectively. Also, by using a Lange skinfold caliper (beta technology Inc, Cambridge, MD USA), body fat percentage (BF \%) was estimated by assessing subcutaneous fat of seven skinfold sites based on Jackson and Pollock's instructions ${ }^{34}$.

\section{Handgrip strength tests}

A hand dynamometer with an adjustable grip (TKK 5101 Grip D; Takey, Tokyo, Japan) was used to assess handgrip strength. Participants performed two attempts with both hands, with the arm fully extended, forming an angle of $30^{\circ}$ with respect to the trunk. The maximum score in kilograms for each hand was recorded, and the mean score of both hands was used in the statistical analyses.

\section{Quality of life}

European Organization for Research and Treatment of Cancer Questionnaire (EORTC- QLQ-C30) developed to assess the quality of life of cancer patients. The validity and reliability of this questionnaire were confirmed in the Iranian cancer population ${ }^{35}$. It consists of 30 questions that assess the global health, symptoms (fatigue, pain, nausea, and vomiting), and functional (physical, role, cognitive, emotional, and social) scales. Higher scores in global health and functional scales and a lower score in symptoms indicate better situations.

\section{Vitamin D supplementation}

Participants in the HVD and Y-HVD groups received VD tablets at 4,000 IU daily, and individuals in the YLVD group consumed 2,000 IU daily.

\section{Yoga protocol}

A female certified yoga coach conducted yoga classes. Participants performed yoga twice a week, with each class lasting around 60-90 minutes for twelve weeks. Exercises were selected from the Hatha yoga style and included Asana (physical postures), pranayama (breath control), and Dyana (meditation). The yoga exercises begin with Pranayama (yoga mudra, Respiratory coordination ), then asana (such as Marjaryasana cycle, Balasana, Hindolasana, Bhujangasana, Setu Bandha, Bitilasana, Surya namaskar, Baddha Konasana, Chakki Chalanasana, Utkatasana, Supta Baddha Konasana, Bhujangasana, kriya cycle, Salabhasana, Ardha Pavana Muktasana, Pavanamuktasana, suptaVakra Asana) and ended with 
dyana (Savasana). In order to monitor the intensity of yoga, the Borg Rating of Perceived Exertion (RPE) scale (6-19 score) was gathered after finishing every workout.

\section{Cytokines assessments}

A medical laboratory expert collected the blood samples in overnight fasting from an antecubital vein into $5 c c$ Ethylenediaminetetraacetic acid (EDTA) tubes. Samples were spun at $3000 \mathrm{rpm}$ in a $4^{\circ} \mathrm{C}$ centrifuge for 10 minutes, and separated serum was stored in a $-20^{\circ} \mathrm{C}$ freezer for later analysis. Specific human enzyme-linked immunosorbent assay [ELISA] kits were used to determine serum level of IL-10, TNF- $a$ and IL-6 [DuoSet ELISA, R\&D Systems, Minneapolis, MN]. The intra- and inter- assay coefficients of variation were less than $8 \%$.

Also, a buffy coat layer was removed using a suspension technic and stored at $-70{ }^{\circ} \mathrm{C}$ freezer. RNA samples were extracted using the total RNA extraction Kit (Takara, Japan), and cDNA synthesis was performed using the Takara cDNA synthesis kit (Takara, Japan) according to the manufacturer's instructions. Real-time PCR was performed using the SYBR Green Master Mix kit (Ampliqon, Denmark). The thermal cycling program was as follows: $94^{\circ} \mathrm{C}$ for 5 min followed by 40 cycles of $95^{\circ} \mathrm{C}$ for $30 \mathrm{~s}, 54$ ${ }^{\circ} \mathrm{C}$ for $45 \mathrm{~s}$, and $72{ }^{\circ} \mathrm{C}$ for $30 \mathrm{~s}$. GAPDH mRNA for the normalization of the gene expression analysis was used. The sequence of PCR primers used for the amplification of the protein-coding genes was as follow: IL-6 forward "GTGAGGAACAAGCCAGAGCA ", IL-6 reverse "TGGCATTTGTGGTTGGGTCA"; IL-10 forward "CTTTAAGGGTTACCTGGGTTGC", IL-10 reverse "CTCACTCATGGCTTTGTAGACAC"; TNF-a forward "CTCCCTCTCATCAGTTCCAT" and TNF-a reverse "CAGTTGGTTGTCTTTGAGATC"; GAPDH forward "CGAGATCCCTCCAAAATCAA" GAPDH reverse "AGGTCAGGTCCACCACTGAC". The fold change expression was calculated using the $2^{-\triangle \Delta C T}$ formula.

\section{Statistical analysis}

We used the Statistical Package of Social Sciences (SPSS, IBM, v19) to analyses row data. Data present by mean \pm standard deviation (SD). Shapiro-Wilk analysis confirms data normality. A paired t-test was used to interpret the within-group difference, and an analysis of covariance (ANCOVA) was used to analyze the effects of interventions on the variables. Pre-test data was considered as a covariate. If significant effects were found, Bonferroni post-hoc tests were done. Data of gene expression changes were analyzed by ANOVA. To define the magnitude and direction of the linear relationship between circulatory markers with anthropometric indicators and QoL indicators, the bivariate Pearson correlation coefficient ( $r$ ) was used on the magnitude of changes. The magnitude of changes was calculated by subtracting post-test values from pre-test values. Effect sizes (ES) were also calculated by the change score divided by the SD of the change score to examine the magnitude of differences while controlling for the influence of the sample size ${ }^{36}$ with 0.2 considered as a small ES, $0.2-0.5$ as a moderate ES, $0.5-0.8$ as a large ES, and $>0.8$ as a very large ES. The changes percentage was calculated by formula:

$C P \%=\frac{(\text { posttest-pretest })}{\text { pretest }} \times 100$.

. The significance level was set at $p \leq 0.05$ for all statistical analyses. 


\section{Results}

In general, the average score of the Borg scale was 8-14. The intensity of initial sessions was low and progressively reached moderate intensity towards the end of the protocol. A 12-week VD supplementation period led to a significant increase in VD levels, and a significant difference was observed between both $H D$ and $Y+H D$ groups and $Y+L D$ group $(F=6.5, p=0.005, n p 2=0.33)$.

The descriptive data of performance and psychological variables are presented in Table 1. There were no significant differences between groups in all indices at baseline $(p>0.05)$. Although there was a substantial decrease in body mass in both groups who performed yoga, there were no significant differences between groups $(F=2.9, p<0.070, n p 2=0.19)$. A 12-week intervention significantly decreased body fat percentage in the Y-HVD and Y-LVD groups compared to the HVD group with a moderate effect size $(F=7.2, p<0.003, n p 2=0.36)$. We observed a significant difference between groups at handgrip strength $(F=8.9, p=0.001, \eta p 2=0.41)$ with a moderate effect size. The Bonferroni post hoc test showed the handgrip strength test was significantly increased in both yoga groups than other groups $(p<0.05)$. 
Table 1

performance and psychological indicators of participants before and after performing intervention

\begin{tabular}{|c|c|c|c|c|c|c|c|}
\hline \multicolumn{2}{|l|}{ Variable } & Group & Pre & Post & $\begin{array}{l}\% \\
\text { change }\end{array}$ & $\begin{array}{l}\mathrm{P} \\
\text { within }\end{array}$ & $\begin{array}{l}\mathrm{P} \\
\text { between }\end{array}$ \\
\hline \multirow{3}{*}{\multicolumn{2}{|c|}{ Body mass (kg) }} & HVD & $\begin{array}{l}73.7 \pm \\
12.7\end{array}$ & $\begin{array}{l}73.5 \pm \\
12.5\end{array}$ & -0.11 & 0.727 & \multirow[t]{3}{*}{0.070} \\
\hline & & Y-LVD & $\begin{array}{l}68.1 \pm \\
11.1\end{array}$ & $\begin{array}{l}67.2 \pm \\
10.7\end{array}$ & -1.31 & 0.030 & \\
\hline & & $\begin{array}{l}\text { Y- } \\
\text { HVD }\end{array}$ & $\begin{array}{l}74.5 \pm \\
10.0\end{array}$ & $73.8 \pm 9.7$ & -1.71 & 0.012 & \\
\hline \multirow{3}{*}{\multicolumn{2}{|c|}{ Body fat percentage (\%) }} & HVD & $37.0 \pm 4.4$ & $36.8 \pm 4.3$ & -0.52 & 0.343 & \multirow[t]{3}{*}{0.003} \\
\hline & & Y-LVD & $37.0 \pm 4.1$ & $35.3 \pm 4.3$ & $-4.67 *$ & 0.001 & \\
\hline & & $\begin{array}{l}\text { Y- } \\
\text { HVD }\end{array}$ & $34.8 \pm 3.3$ & $33.4 \pm 2.9$ & $-3.94^{\star}$ & 0.003 & \\
\hline \multirow{3}{*}{\multicolumn{2}{|c|}{$\begin{array}{l}\text { Handgrip strength tests } \\
(\mathrm{kg})\end{array}$}} & HVD & $18.0 \pm 4.3$ & $18.3 \pm 4.3$ & 1.73 & 0.345 & \multirow[t]{3}{*}{0.001} \\
\hline & & Y-LVD & $16.4 \pm 2.8$ & $18.4 \pm 2.4$ & $13.32^{\star}$ & 0.001 & \\
\hline & & $\begin{array}{l}\text { Y- } \\
\text { HVD }\end{array}$ & $18.7 \pm 4.0$ & $20.0 \pm 4.3$ & $7.09 *$ & 0.001 & \\
\hline \multirow{3}{*}{\multicolumn{2}{|c|}{ Vitamin D (IU) }} & $\mathrm{HD}$ & $\begin{array}{l}41.2 \pm \\
16.2\end{array}$ & $\begin{array}{l}53.5 \pm \\
15.9\end{array}$ & 34.62 & 0.001 & \multirow[t]{3}{*}{0.005} \\
\hline & & $\begin{array}{l}Y+ \\
\mathrm{HD}\end{array}$ & $\begin{array}{l}44.8 \pm \\
13.1\end{array}$ & $\begin{array}{l}57.5 \pm \\
12.3\end{array}$ & $32.17 \#$ & 0.001 & \\
\hline & & $\begin{array}{l}Y+ \\
\text { LD }\end{array}$ & $\begin{array}{l}43.4 \pm \\
15.1\end{array}$ & $\begin{array}{l}49.3 \pm \\
16.2\end{array}$ & $15.69 *$ & 0.001 & \\
\hline \multirow{6}{*}{$\begin{array}{l}\text { Quality of life } \\
\text { questionnaire }\end{array}$} & \multirow[t]{3}{*}{ Global health } & HVD & $\begin{array}{l}70.8 \pm \\
21.2\end{array}$ & $\begin{array}{l}69.2 \pm \\
22.2\end{array}$ & -3.01 & 0.161 & \multirow[t]{3}{*}{0.001} \\
\hline & & Y-LVD & $\begin{array}{l}58.3 \pm \\
14.7\end{array}$ & $\begin{array}{l}75.8 \pm \\
15.4\end{array}$ & $33.78^{*}$ & 0.001 & \\
\hline & & $\begin{array}{l}\text { Y- } \\
\text { HVD }\end{array}$ & $\begin{array}{l}59.9 \pm \\
17.5\end{array}$ & $\begin{array}{l}82.5 \pm \\
12.7\end{array}$ & $47.01^{*}$ & 0.001 & \\
\hline & \multirow[t]{3}{*}{$\begin{array}{l}\text { functional } \\
\text { scales }\end{array}$} & HVD & $\begin{array}{l}68.4 \pm \\
18.2\end{array}$ & $\begin{array}{l}70.7 \pm \\
19.5\end{array}$ & 3.38 & 0.351 & \multirow[t]{3}{*}{0.001} \\
\hline & & Y-LVD & $\begin{array}{l}58.4 \pm \\
19.8\end{array}$ & $81.1 \pm 9.8$ & $49.9 *$ & 0.001 & \\
\hline & & $\begin{array}{l}\text { Y- } \\
\text { HVD }\end{array}$ & $\begin{array}{l}55.4 \pm \\
17.9\end{array}$ & $84.2 \pm 7.9$ & $62.8^{*}$ & 0.001 & \\
\hline
\end{tabular}




\begin{tabular}{|c|c|c|c|c|c|c|c|}
\hline \multicolumn{2}{|l|}{ Variable } & Group & Pre & Post & $\begin{array}{l}\% \\
\text { change }\end{array}$ & $\begin{array}{l}\mathrm{P} \\
\text { within }\end{array}$ & $\begin{array}{l}\mathrm{P} \\
\text { between }\end{array}$ \\
\hline & \multirow[t]{3}{*}{$\begin{array}{l}\text { symptom } \\
\text { scales }\end{array}$} & HVD & $\begin{array}{l}34.8 \pm \\
13.7\end{array}$ & $\begin{array}{l}34.5 \pm \\
12.6\end{array}$ & 3.85 & 0.928 & \multirow[t]{3}{*}{0.001} \\
\hline & & Y-LVD & $\begin{array}{l}41.8 \pm \\
18.8\end{array}$ & $\begin{array}{l}26.2 \pm \\
11.6\end{array}$ & $-35.0 *$ & 0.001 & \\
\hline & & $\begin{array}{l}\text { Y- } \\
\text { HVD }\end{array}$ & $\begin{array}{l}43.4 \pm \\
12.2\end{array}$ & $18.6 \pm 4.8$ & $-54.1^{\star}$ & 0.005 & \\
\hline
\end{tabular}

HVD: High dose of vitamin D, Y-LVD: yoga with a low dose of vitamin D, Y-HVD: yoga with a high dose of vitamin $D$. *significant difference with HVD group. \# Significant difference with $Y$-LVD group

We observed significant differences in the Qol questionnaire between groups at global health $(F=15.0, p$ $<0.001, \eta p 2=0.54)$, functional scales $(F=12.9, p<0.001, \eta p 2=0.49)$, and symptoms scales $(F=13.0, p<$ $0.001, n p 2=0.50)$ with a moderate effect size. Three months of yoga classes effectively improved the Qol in both the Y-HVD and Y-LVD groups compared to the HVD group (Table 1).

There were no significant differences in the circulatory level of IL-6 between groups $(F=1.2, p=0.318$, $\eta p 2=0.08)$. Also, in intra-group changes, there was a substantial decrease in the Y-HVD group $(p=$ $0.001,-30.9 \%)$, but there were no significant changes in the Y-LVD $(p=0.150,-16.3 \%$ and HVD group $(p=$ $0.390,-10.2 \%)$ (Fig. 1a).

Although there were significant decreases in the circulatory level of TNF-a in the Y-LVD $(p=0.034,-13.0 \%)$ and Y-HVD groups ( $p=0.001,-24.7 \%)$, not in the HVD group $(p=0.149,-8.7 \%)$, these changes were not significant between groups $(F=1.6, p<0.230, n p 2=0.11)(F i g .1 b)$.

There was a significant difference in the circulatory level of IL-10 between groups $(F=5.7, p<0.009, \eta p 2$ $=0.31$ ) with a moderate effect size. The Bonferroni post hoc test showed the difference was between the Y-HVD group with the other groups $(p<0.05)$. Serum concentration of IL10 significantly increased in the Y-HVD $(p=0.001,61.9 \%)$, the Y-LVD $(p=0.023,19.4 \%)$, and the HVD groups $(p=0.025,17.0 \%)(F i g .1 c)$.

The ratio of IL-10/ TNF-a was measured as an anti-inflammatory index. There was a significant difference in the IL-10/ TNF-a ratio between groups $(F=9.3, p<0.001, \eta p 2=0.42)$ with a moderate effect size. The Bonferroni post hoc test showed changes in the Y-HVD group differ significantly from the other groups $(p<0.05)$. In intra-group changes, there were significant increases in the Y-HVD group $(p=0.001$, $117.4 \%)$, the HVD $(p=0.021,36.6 \%)$ and Y-LVD $(p=0.030,48.8 \%)$ groups (Fig. $1 d)$.

Table 2 presents the correlations between circulatory markers and weight, BF\%, strength, and QoL indicators. There were significant negative relations between IL-10 changes and changes in weight $(r=-0.51), B F \%(r=-0.38)$, and positive association with global health on of the QoL indicator $(r=0.52)$. TNF-a and IL- 6 changes only demonstrated a significant positive correlation with BF\% (Table 2). 
Significant negative correlation setween IL-10/ TNF-a ratio changes and weight $(\mathrm{r}=-0.38)$ and BF\% $(r=-0.59)$ changes and significant positive global health changes $(r=0.42)$ were observed (Table 2$)$.

Table 2

correlation between changes in inflammatory markers and weight, body fat\%, and quality of life indicator after the intervention.

\begin{tabular}{|lllllll|}
\hline Variables & $\Delta$ Weight & $\Delta$ BFP & $\Delta$ HGS & $\Delta$ GH & $\Delta$ FS & $\Delta$ SS \\
\hline$\Delta$ IL-6 & 0.15 & $0.43^{*}$ & -0.03 & -0.24 & 0.04 & 0.17 \\
\hline$\Delta$ TNF-a & 0.03 & $0.36^{*}$ & -0.13 & -0.13 & -0.01 & 0.04 \\
\hline$\Delta$ IL-10 & $-0.51^{*}$ & $-0.38^{*}$ & 0.19 & $0.52 *$ & 0.34 & -0.33 \\
\hline$\Delta$ IL-10/ TNF-a ratio & $-0.38^{*}$ & $-0.59 *$ & 0.28 & $0.42^{*}$ & 0.17 & -0.24 \\
\hline
\end{tabular}

BFP: Body Fat Percentage; HGS: handgrip strength; GH: Global Health; FS: Functional Scales; SS: Symptom Scales; * significant correlation $(p<0.05)$

Figure 2a shows the changes in IL- 6 gene expression in the groups after intervention. A significant difference was observed in leukocyte's IL-6 expression between the groups $(F=3.8, p=0.034)$. The Bonferroni post hoc test showed the significant difference was between the HVD group and Y-HVD. IL-6 expression upregulated in the HVD; doing yoga led to a decline in IL-6 expression (Fig. 2a).

Following the intervention, there were no significant differences in leukocytes TNF-a expression between groups $(F=3.39, p=0.075)$ (Fig. $2 b)$.

In addition, a significant difference was observed in leukocyte's IL-10 expression between groups after intervention ( $F=3.80, p=0.036)$. Expression of IL-10 increased in all groups (Fig. 7); the magnitude of the increase has significantly differed in the Y-HVD group and the Y-LVD group (Fig. 2c).

Interventions enhanced the anti-inflammatory index, IL-10/ TNF-a ratio, in the peripheral mononuclear cells. There were significant differences in the IL-10/ TNF-a ratio between groups $(F=23.5, p<0.001)$. The Bonferroni post hoc test showed increases in the Y-HVD group (32.4\%) differ significantly from the Y-LVD (11.2\%) and the HVD (9.2\%) groups (Fig. 2d).

\section{Discussion}

The primary aim of this study was to evaluate the effectiveness of adding 12 weeks of yoga training to high VD dose on cytokine profile and Qol in BC survivors. Also, possible relations between cytokine levels with functional and psychological indices were assessed. High VD dose supplementations led to significant increases in VD level than low VD dose. The findings indicate the high amount of VD alone did not significantly improve the systemic inflammation and QoL and performance indicators. Still, substantial improvements in Qol, handgrip strength, and body composition were observed in combination with yoga. Moreover, yoga with a high VD dose led to marked increases in the circulatory level of IL-10 and decreases in the concentrations of IL-6 and TNF-a. Also, the anti-inflammatory index was increased 
in the yoga plus high VD dose group. These results were relatively parallel with changes in inflammatory cytokines gene expression in peripheral blood cells. In addition, there were significant correlations between circulatory markers changes, especially IL-10 and IL-10/TNF-a ratio changes, and weight and $\mathrm{BF} \%$ changes as well as global health of QoL indicators. Moreover, our findings indicated that combination of yoga and low dose of VD improved QoL, handgrip strength, and body composition but did not show synergistic effects on cytokine balance in genes expression level in peripheral blood cells and plasma level.

The findings supported previous research demonstrating that yoga improves BC survivors' QoL ${ }^{37,38}$. In this regard, Vadiraja et al. (2009), in a randomized controlled trial study, showed that six weeks of yoga significantly improved emotional function, cognitive function and reduced adverse effects, which in total led to an improvement in the QoL of BC patients undergoing radiotherapy ${ }^{38}$. Several mechanisms are proposed for improving the QoL with yoga, including promoting physical fitness and independence, improving social behaviors and the feeling of empathy resulting from group training, and reducing anxiety ${ }^{39}$. Interestingly, the improved QoL coincided with improving body composition and handgrip strength and boosting the immune system by lowering systemic inflammation.

Cancer-related fatigue and its treatments are associated with low activity, result in adverse effects on body composition and muscle atrophy, leading to a decline in BC survivors' QoL. Elevated body mass via increased BF\% could intensify sedentary behaviors and causes complications such as metabolic diseases. On the other hand, exercise interventions like yoga can increase energy expenditure as long as the energy intake is constant, leading to losing weight by fat burning and positively affecting body composition ${ }^{40-43}$. Our finding supported previous studies and showed that a 12-week yoga practice period reduced BF\% by $4.7 \%$ and $3.9 \%$ in the Y-LVD and Y-HVD groups, respectively. In addition, decreased muscle strength is another complication of sedentary behaviors. It was reported that handgrip strength might be an important correlate of health in BC survivors and could be an adjuvant method for evaluation function abilities ${ }^{44}$. We observed a marked increase in the handgrip strength in both Y-HVD (7.1\%) and Y-LVD (13.3\%) groups. In this regard, substantial improvements in handgrip strength have been reported in patients with rheumatoid arthritis ${ }^{45}$ and the affected side in BC patients ${ }^{46}$ with yoga exercises. Yoga movements include stretching and muscular endurance exercises that can inherently enhance strength by promoting neuromuscular coordination, especially in those who have experienced extreme muscle weakness. Therefore, yoga as an exercise intervention is effective in lowering $\mathrm{BF} \%$ and improving muscle strength.

Improved the QoL of the participants was associated with a reduction in systemic inflammation indicators and an improvement in the anti-inflammatory index. Following the intervention, the IL- 6 gene expression in PBMCs increased in the HVD group, but yoga leads to decreased it in both Y-LVD and YHVD groups; serum concentrations of IL- 6 were reduced in all groups, but only it was significantly reduced in the Y-HVD group (30\%). In this regard, Long parma et al. (2015) also stated that six months of yoga did not affect inflammatory serum markers such as IL-6, IL-8, and TNF-a ${ }^{43}$. In contrast, some researchers 
reported yoga could lower inflammatory markers, IL-6, and TNF-a $25,47,48$. Although little is known about mechanisms underlying reducing inflammatory markers with yoga, reducing $\mathrm{BF} \%$ and increasing in parasympathetic nervous system via the anti-inflammatory cholinergic pathway ${ }^{49}$ lead to reduce inflammation. The observed significant positive correlation $(r=0.43)$ between IL- 6 changes and BF\% changes approved that reducing fat tissue with the combination of yoga with low and high VD doses were involved in decreasing IL-6 levels. In addition, given most BC survivors report problems with sleep 50 and sleep disturbance can activate inflammatory signaling, improve sleep quality with yoga might account for lowering inflammatory markers in BC survivors. On the other hand, VD can suppress the production of pro-inflammatory cytokine, IL-6, through inhibition of p38 activation and cytokine production in leukocytes ${ }^{51}$, as well as down-regulation of NF-KB expression in human lymphocytes ${ }^{52}$, thus, the greatest impact on reducing IL- 6 was seen in the Y-HVD group.

Also, we observed a significant increase in the anti-inflammatory index, the IL-10/ TNF-a ratio, in the YHVD group compared to other groups following the intervention in the serum and gene expression levels. This change was due to a marked increase in an anti-inflammatory cytokine, IL-10, and a non-significant decrease in a pro-inflammatory cytokine, TNF-a. In this regard, researchers reported no significant changes in serum TNF-a levels after 10-12 weeks of yoga training $47,48,53$ and a considerable increase in serum IL-10 levels after 12 weeks of yoga ${ }^{54}$. Researchers have attributed these improvements to the antiinflammatory effects of exercise training ${ }^{55}$ and to burn fat tissue ${ }^{42}$. Researchers reported that increased production of IL-10, an anti-inflammatory cytokine, leads to inhibition of the synthesis of proinflammatory cytokines, including TNF-a as well as its receptors ${ }^{56,57}$. Also, the observed substantial correlations between IL-10 ( $r=-0.38)$ and TNF- $a(r=0.36)$ changes and BF\% prove that a decrease in adipose tissue is involved at serum levels of these cytokines. Moreover, VD could suppress TNF- $a$ production through inhibition of p38 activation ${ }^{51}$ and down-regulation of NF-KB expression in human leukocytes ${ }^{52}$ and regulates the IL-10 gene expression in human B cells, increasing serum IL-10 level ${ }^{58}$. In this study, a further increase in IL-10 gene expression in the HVD (2.6 fold) and Y-HVD (4.4 fold) groups compared to the Y-LVD group ( 1.8 fold) indicated that high VD dose has obvious anti-inflammatory effects. On the other hand, it seems that the degree of adaptations is intensity-dependent. Given that moderate-intensity aerobic training led to a greater reduction in TNF-a levels than low-intensity exercise 59 , it might need to perform yoga with higher intensity for a significant effect.

We acknowledge that there are some limitations in this study. Firstly, monitoring the participants' diets and determining the amount of VD intake from the diet was difficult because of the intervention duration. Secondly, individualizing the intensity of yoga in a group class is very difficult and complicated; however, we used the Borg scale, representing the degree of the mental and physical difficulty of the work done. Given that the intensity may be an essential factor in the concentration of inflammatory cytokines, we recommend that the effect of exercise intensity be investigated in future research. In addition, we recommend that the investigation be conducted over a longer length, and the participants would be followed up to determine the persistence of the adaptations. 


\section{Conclusions}

Totally, receiving high doses of VD is safe and effective in reducing inflammatory markers. Also, executing yoga effectively improves the physical and mental status and QoL of BC survivors. Therefore, combining both approaches, yoga with a high VD dose, is a practical behavioral approach to equilibrate anti- and pro-inflammatory markers and promote physical and mental fitness in cancer survivors.

\section{Abbreviations}

BC: Breast cancer

Qol: Quality of life

IL-6: Interleukin-6

IL-10: Interleukin-10

TNF-a: Tumor necrosis factor-a

PBMCs: Peripheral blood mononuclear cells

VD: Vitamin D

HVD: High dose of VD

Y-HVD: Yoga with a high dose of VD

Y-LVD: Yoga with a low dose of VD

$\mathrm{BF} \%$ : Body fat percentage

RPE: Rating of Perceived Exertion

ANCOVA: Analysis of covariance

ES: Effect sizes

\section{Declarations}

\section{Availability of data and materials}

Data would be available from the corresponding author on reasonable request.

Acknowledgments 
We want to acknowledge all participants and a yoga teacher of Payammehr yoga center who collaborated with us.

\section{Conflict of interest}

The authors declare that they have no conflict of interest.

\section{Funding}

There was no funding support

\section{Ethics approval and consent to participate}

The ethics committee of Sport Sciences Research Institute of Iran (approval number: IR.SSRI.REC.1398.111) approved all research procedures, and this study was conducted under the Declaration of Helsinki. After being informed of the benefits and risks of research, participants signed written consent.

\section{Author contributions}

S-AS, MK, and MM-S designed the study. MN, HK, ZA, and VKZ conducted the intervention. SA-S analyzed the data. MM-S, S-AS and MK interpreted the data for the study. SA-S, MK and MM-S wrote the first draft, and JW revised it. All authors read and approved the final version of manuscript.

\section{References}

1. Lovelace DL, McDaniel LR, Golden D. Long-term effects of breast cancer surgery, treatment, and survivor care. J Midwifery Women Health. 2019;64:713-24.

2. Tsaras K, Papathanasiou IV, Mitsi D, et al. Assessment of depression and anxiety in breast cancer patients: Prevalence and associated factors. Asian Pacific journal of cancer prevention: APJCP. 2018;19:1661-9.

3. Bower JE, Ganz PA, Irwin MR, et al. Fatigue and gene expression in human leukocytes: Increased nf$\mathrm{Kb}$ and decreased glucocorticoid signaling in breast cancer survivors with persistent fatigue. Brain Behav Immun. 2011;25:147-50.

4. Bower JE, Ganz PA, Aziz N, et al. Fatigue and proinflammatory cytokine activity in breast cancer survivors. Psychosom Med. 2002;64:604-11.

5. Kozłowski L, Zakrzewska I, Tokajuk P, et al: Concentration of interleukin-6 (il-6), interleukin-8 (il-8) and interleukin-10 (il-10) in blood serum of breast cancer patients. Roczniki Akademii Medycznej w Bialymstoku (1995) 2003, 48:82 - 4.

6. Berberoglu U, Yildirim E, Celen O. Serum levels of tumor necrosis factor alpha correlate with response to neoadjuvant chemotherapy in locally advanced breast cancer. Int J Biol Mark. 2004;19:130-4. 
7. Hamidullah CB, Konwar R. Role of interleukin-10 in breast cancer. Breast Cancer Res Treat. 2012;133:11-21.

8. Molanouri Shamsi M, Chekachak S, Soudi S, et al. Combined effect of aerobic interval training and selenium nanoparticles on expression of il-15 and il-10/tnf-a ratio in skeletal muscle of $4 \mathrm{t} 1$ breast cancer mice with cachexia. Cytokine. 2017;90:100-8.

9. Kumari R, Kumar S, Ahmad MK, et al. Tnf-a/il-10 ratio: An independent predictor for coronary artery disease in north indian population. Diabetes Metabolic Syndrome: Clinical Research Reviews. 2018;12:221-5.

10. Connolly PH, Caiozzo VJ, Zaldivar F, et al.: Effects of exercise on gene expression in human peripheral blood mononuclear cells. Journal of applied physiology (Bethesda, Md: 1985) 2004, 97:1461-9.

11. Brenner DR, Scherer D, Muir K, et al.: A review of the application of inflammatory biomarkers in epidemiologic cancer research. Cancer epidemiology, biomarkers \& prevention: a publication of the American Association for Cancer Research, cosponsored by the American Society of Preventive Oncology 2014, 23:1729-51.

12. Andersen MR, Sweet E, Hager S, et al. Effects of vitamin d use on health-related quality of life of breast cancer patients in early survivorship. Integrative cancer therapies. 2019;18:1534735418822056-6.

13. Peppone LJ, Rickles AS, Janelsins MC, et al. The association between breast cancer prognostic indicators and serum 25-oh vitamin d levels. Ann Surg Oncol. 2012;19:2590-9.

14. Goodwin PJ, Ennis M, Pritchard KI, et al. Prognostic effects of 25-hydroxyvitamin d levels in early breast cancer. Journal of clinical oncology: official journal of the American Society of Clinical Oncology. 2009;27:3757-63.

15. Liu W, Zhang L, Xu HJ, et al.: The anti-inflammatory effects of vitamin d in tumorigenesis. Int J Mol Sci 2018, 19.

16. Nonn L, Peng L, Feldman D, et al. Inhibition of p38 by vitamin d reduces interleukin-6 production in normal prostate cells via mitogen-activated protein kinase phosphatase 5: Implications for prostate cancer prevention by vitamin d. Cancer Res. 2006;66:4516-24.

17. Boonstra A, Barrat FJ, Crain C, et al.: 1alpha,25-dihydroxyvitamin d3 has a direct effect on naive cd4(+) t cells to enhance the development of th2 cells. Journal of immunology (Baltimore, Md: 1950) 2001, 167:4974-80.

18. Barrat FJ, Cua DJ, Boonstra $A$, et al. In vitro generation of interleukin 10-producing regulatory cd4(+) $t$ cells is induced by immunosuppressive drugs and inhibited by thelper type 1 (th1)- and th2-inducing cytokines. J Exp Med. 2002;195:603-16.

19. Fisher SA, Rahimzadeh M, Brierley $\mathrm{C}$, et al. The role of vitamin d in increasing circulating t regulatory cell numbers and modulating t regulatory cell phenotypes in patients with inflammatory disease or in healthy volunteers: A systematic review. PLoS One. 2019;14:e0222313. 
20. Yu Y, Tian L, Xiao Y, et al. Effect of vitamin d supplementation on some inflammatory biomarkers in type 2 diabetes mellitus subjects: A systematic review and meta-analysis of randomized controlled trials. Ann Nutr Metab. 2018;73:62-73.

21. Häusler D, Torke $S$, Peelen E, et al. High dose vitamin d exacerbates central nervous system autoimmunity by raising t-cell excitatory calcium. Brain. 2019;142:2737-55.

22. Bower JE, Woolery A, Sternlieb B, et al. Yoga for cancer patients and survivors. Cancer control: journal of the Moffitt Cancer Center. 2005;12:165-71.

23. Riley D. Hatha yoga and the treatment of illness. Alternative therapies in health and medicine 2004, $10: 20-1$.

24. Dai S, Mo Y, Wang Y, et al. Chronic stress promotes cancer development. Front Oncol. 2020;10:14922.

25. Kiecolt-Glaser JK, Christian L, Preston H, et al. Stress, inflammation, and yoga practice. Psychosom Med. 2010;72:113-21.

26. Jong MC, Boers I, Schouten van der Velden AP, et al.: A randomized study of yoga for fatigue and quality of life in women with breast cancer undergoing (neo) adjuvant chemotherapy. Journal of alternative and complementary medicine (New York, NY) 2018, 24:942-953.

27. Balaji P, Varne SR, Ali SS. Physiological effects of yogic practices and transcendental meditation in health and disease. North American journal of medical sciences. 2012;4:442.

28. Taso C-J, Lin H-S, Lin W-L, et al. The effect of yoga exercise on improving depression, anxiety, and fatigue in women with breast cancer: A randomized controlled trial. Journal of Nursing Research. 2014;22:155-64.

29. Bernardi L, Sleight P, Bandinelli G, et al. Effect of rosary prayer and yoga mantras on autonomic cardiovascular rhythms: Comparative study. Bmj. 2001;323:1446-9.

30. Kiecolt-Glaser JK, Christian L, Preston H, et al. Stress, inflammation, and yoga practice. Psychosom Med. 2010;72:113-21.

31. !!! INVALID CITATION !!! [8].

32. Molanouri Shamsi M, Hassan ZM: Chap. 42 - exercise, selenium, and cancer cells. In Cancer (second edition). Edited by Preedy VR and Patel VB. San Diego: Academic Press; 2021:475-482.

33. Crew KD, Xiao T, Thomas PS, et al.: Safety, feasibility, and biomarker effects of high-dose vitamin d supplementation among women at high risk for breast cancer. International journal of food science, nutrition and dietetics 2015, 2015:1-16.

34. Jackson AS, Pollock ML, Ward A. Generalized equations for predicting body density of women. Med Sci Sports Exerc. 1980;12:175-81.

35. Montazeri A, Harirchi I, Vahdani M, et al. The european organization for research and treatment of cancer quality of life questionnaire (eortc qlq-c30): Translation and validation study of the iranian version. Support Care Cancer. 1999;7:400-6. 
36. Dankel SJ, Loenneke JP. Effect sizes for paired data should use the change score variability rather than the pre-test variability. J Strength Cond Res 2018.

37. Vardar Yağıı N, Şener G, Arıkan H, et al. Do yoga and aerobic exercise training have impact on functional capacity, fatigue, peripheral muscle strength, and quality of life in breast cancer survivors? Integrative Cancer Therapies. 2015;14:125-32.

38. Vadiraja HS, Rao MR, Nagarathna R, et al. Effects of yoga program on quality of life and affect in early breast cancer patients undergoing adjuvant radiotherapy: A randomized controlled trial. Complement Ther Med. 2009;17:274-80.

39. Ülger Ö, Yağlı NV. Effects of yoga on the quality of life in cancer patients. Complementary Therapies in Clinical Practice. 2010;16:60-3.

40. Hughes DC, Darby N, Gonzalez K, et al. Effect of a six-month yoga exercise intervention on fitness outcomes for breast cancer survivors. Physiother Theory Pract. 2015;31:451-60.

41. Littman AJ, Bertram LC, Ceballos R, et al. Randomized controlled pilot trial of yoga in overweight and obese breast cancer survivors: Effects on quality of life and anthropometric measures. Support Care Cancer. 2012;20:267-77.

42. Cramer $H$, Thoms MS, Anheyer $D$, et al. Yoga in women with abdominal obesity-a randomized controlled trial. Deutsches Ärzteblatt International. 2016;113:645.

43. Long Parma D, Hughes DC, Ghosh $S$, et al. Effects of six months of yoga on inflammatory serum markers prognostic of recurrence risk in breast cancer survivors. SpringerPlus. 2015;4:143.

44. Cantarero-Villanueva I, Fernandez C, Diaz Rodriguez L, et al. The handgrip strength test as a measure of function in breast cancer survivors relationship to cancer-related symptoms and physical and physiologic parameters. Am J Phys Med Rehabil/Assoc Acad Physiatr. 2012;91:774-82.

45. Dash $\mathrm{M}$, Telles $\mathrm{S}$. Improvement in hand grip strength in normal volunteers and rheumatoid arthritis patients following yoga training. Indian J Physiol Pharmacol. 2001;45:355-60.

46. Mazor M, Lee JQ, Peled A, et al. The effect of yoga on arm volume, strength, and range of motion in women at risk for breast cancer-related lymphedema. The Journal of Alternative Complementary Medicine. 2018;24:154-60.

47. Kiecolt-Glaser JK, Bennett JM, Andridge R, et al. Yoga's impact on inflammation, mood, and fatigue in breast cancer survivors: A randomized controlled trial. Journal of clinical oncology: official journal of the American Society of Clinical Oncology. 2014;32:1040-9.

48. Bower JE, Greendale G, Crosswell AD, et al. Yoga reduces inflammatory signaling in fatigued breast cancer survivors: A randomized controlled trial. Psychoneuroendocrinology. 2014;43:20-9.

49. Tracey KJ. Reflex control of immunity. Nat Rev Immunol. 2009;9:418-28.

50. Savard J, Simard S, Blanchet J, et al. Prevalence, clinical characteristics, and risk factors for insomnia in the context of breast cancer. Sleep. 2001;24:583-90.

51. Zhang Y, Leung DY, Richers BN, et al.: Vitamin d inhibits monocyte/macrophage proinflammatory cytokine production by targeting mapk phosphatase-1. Journal of immunology (Baltimore, Md: 
1950) 2012, 188:2127-35.

52. Geldmeyer-Hilt K, Heine G, Hartmann B, et al. 1,25-dihydroxyvitamin d3 impairs nf-kb activation in human naïve b cells. Biochem Biophys Res Commun. 2011;407:699-702.

53. Nugent NR, Brick L, Armey MF, et al.: Benefits of yoga on il-6: Findings from a randomized controlled trial of yoga for depression. Behavioral medicine (Washington, DC) 2019:1-10.

54. Rajbhoj PH, Shete SU, Verma A, et al. Effect of yoga module on pro-inflammatory and antiinflammatory cytokines in industrial workers of lonavla: A randomized controlled trial. Journal of clinical diagnostic research: JCDR. 2015;9:CC01.

55. Bruunsgaard $\mathrm{H}$. Physical activity and modulation of systemic low-level inflammation. J Leukoc Biol. 2005;78:819-35.

56. Braun DA, Fribourg M, Sealfon SC. Cytokine response is determined by duration of receptor and signal transducers and activators of transcription 3 (stat3) activation. J Biol Chem. 2013;288:298693.

57. Starkie R, Ostrowski SR, Jauffred S, et al. Exercise and il-6 infusion inhibit endotoxin-induced tnf-a production in humans. FASEB J. 2003;17:1-10.

58. Heine G, Niesner U, Chang HD, et al. 1, 25-dihydroxyvitamin d3 promotes il-10 production in human $b$ cells. Eur J Immunol. 2008;38:2210-8.

59. Abd El-Kader S, Gari A, El-Den S. A: Impact of moderate versus mild aerobic exercise training on inflammatory cytokines in obese type 2 diabetic patients: A randomized clinical trial. Afr Health Sci. 2013;13:857-63.

\section{Figures}


A
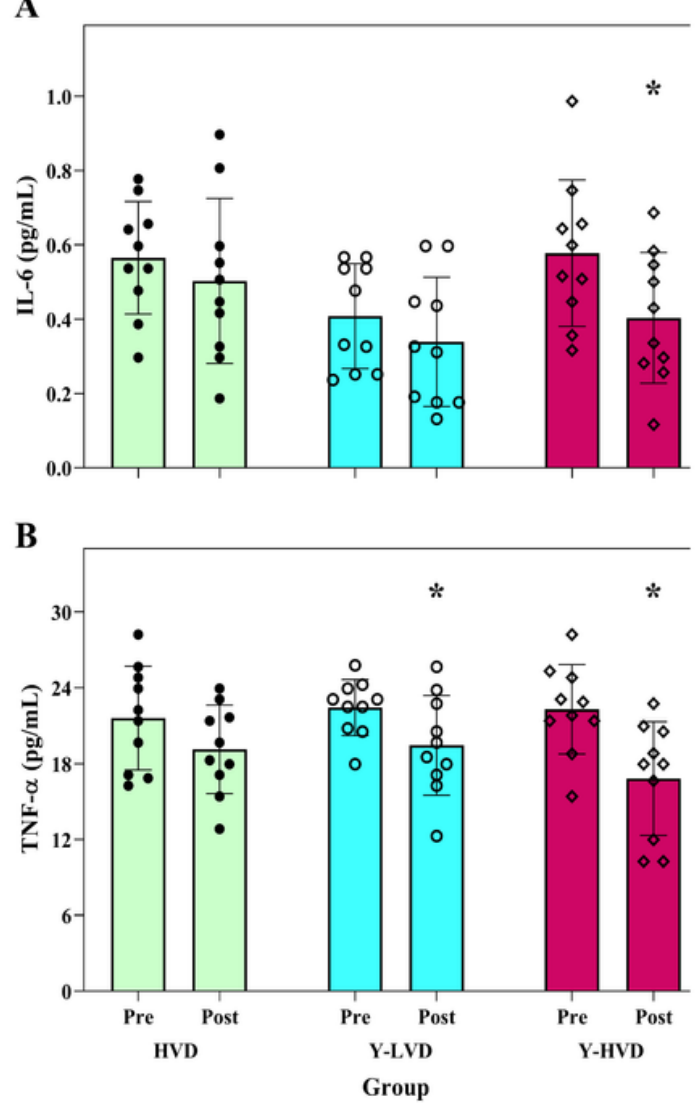

$\mathrm{C}$

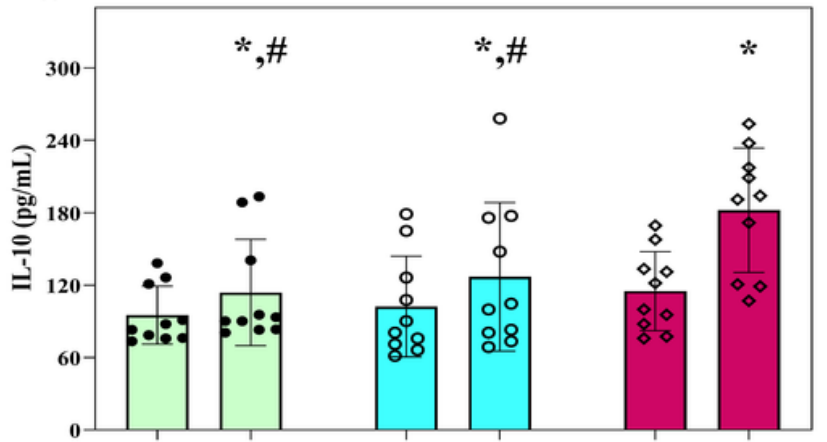

$\mathrm{D}$

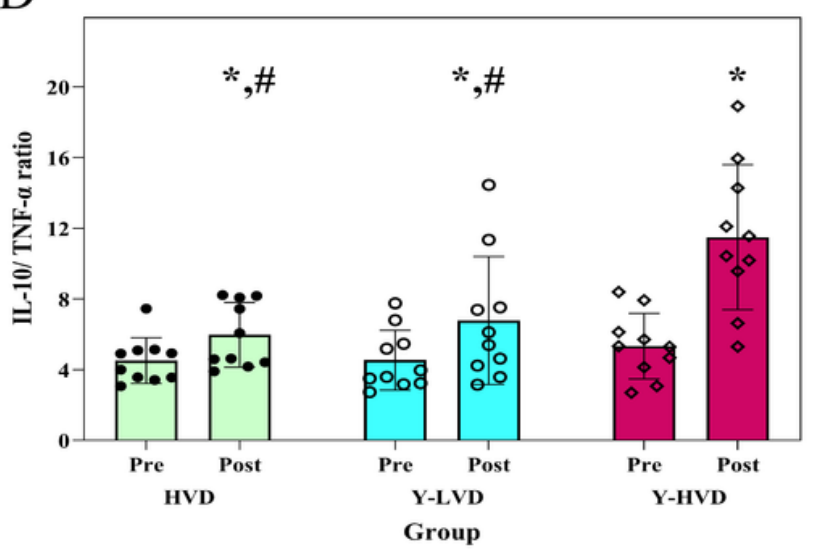

Figure 1

Concentration of serum IL-6 (a), TNF-a (b), IL-10 (c) and IL-10/TNF-a ratio (d) at baseline and after intervention. HVD: a high dose of vitamin D, Y-LVD: yoga with a low dose of vitamin D, Y-HVD: yoga with a high dose of vitamin $\mathrm{D}$. *Significant difference from pre-to post intervention; \#significant difference with the Y-HVD group. 

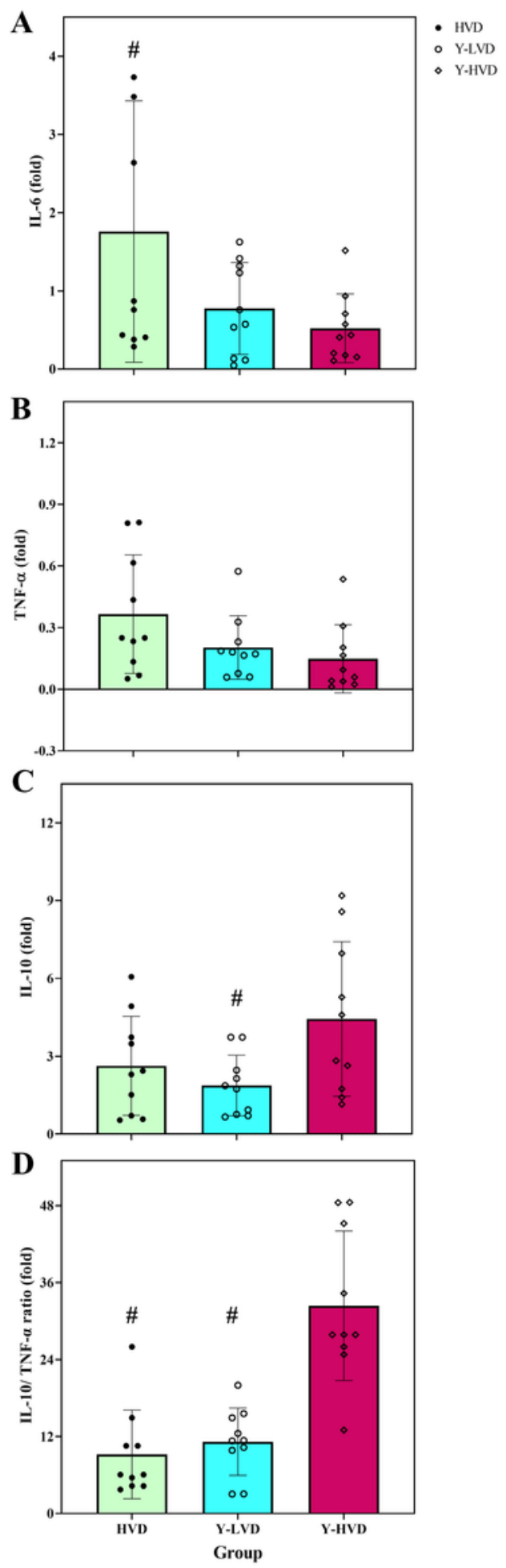

\section{Figure 2}

Gene expression of IL-6 (a), TNF-a (b), IL-10 (c) and IL-10/TNF-a ratio (d) at baseline and after intervention in peripheral blood cells. HVD: a high dose of vitamin D, Y-LVD: yoga with a low dose of vitamin D, Y-HVD: yoga with a high dose of vitamin D. *Significant difference from pre-to post intervention; \#significant difference with the Y-HVD group. 